\title{
Preparation of highly stable polypyrrole dispersions in the mixed aqueous solution of sodium dodecyl sulfate and polyvinyl pyrrolidone
}

\author{
Shuangxi Xing, * Guoku Zhao \\ Faculty of Chemistry, Northeast Normal University, Changchun, 130024, P. R. China. \\ E-mail:xingsx737@nenu.edu.cn.
}

(Received: 28 May, 2007; published: 17 August, 2007)

\begin{abstract}
Polypyrrole (PPy) dispersions with high stability were prepared in the mixed aqueous solution comprising an anionic surfactant (sodium dodecyl sulfate, SDS) and a polymeric stabilizer (polyvinyl pyrrolidone, PVP). By choosing proper mass ratios of the surfactant to the monomer, stable dispersions could be obtained owing to the interaction between the two components. The structure, morphology, stability, particle size, polydispersity index and conductivity of the samples obtained from different conditions were investigated. The PPy dispersions prepared from mixed aqueous solutions were much more stable than those from single component solution.

Keywords: polypyrrole; dispersions; sodium dodecyl sulfate; polyvinyl pyrrolidone
\end{abstract}

\section{Introduction}

Polypyrrole (PPy) as one of the most important conducting polymers has received comprehensive interests in recent years [1-4]. It has such merits as easy preparation, environmental stability and high conductivity, which leads to its wide applications in various fields such as sensors, actuators and electric devices. However, the poor solubility in most solvents limits its processability and hence its application. The preparation of PPy in its colloidal forms has been sought to improve its processability. Therefore, many kinds of steric stabilizers including poly(vinyl pyrrolidone) (PVP) [5], poly(ethylene oxide) (PEO) [6], poly(styrene sulfonate)(PSS) [7], poly(vinyl methyl ether)(PVME) [8,9], ethylhydroxycellulose [10] and so on have already been used to prepare PPy dispersions. Copolymers like poly $(\mathrm{N}$ isopropylacrylamide-co-acrylamide) is also a candidate for stabilizing PPy dispersion [11]. Besides, spherical and "stringy" silica have been utilized for the preparation of PPy dispersions [12]. Among the methods mentioned above, Andrij Pich et al. [13] have studied the PPy dispersions using PVME as stabilizer and they also discussed the effect of three different anionic salts as dopants on the properties of the resulting dispersions. S.P. Armes et al. [14] have reported the preparation of colloidal dispersions of surfactant-stabilized PPy particles using sodium dodecylbenzene sulfonate (SDBS) and ammonium persulfate (APS) as surfactant and oxidant, respectively. In our former experiments, we have prepared PPy dispersions using SDBS as surfactant and investigated their stability and particle size in detail [15]. However, there still exist many problems that should be resolved, especially the improvement of the stability of the PPy dispersions, which could greatly widen the application of the PPy. To this aim, we tried the preparation of PPy dispersion in the mixed aqueous solution comprising sodium dodecyl sulfate (SDS) and PVP, because 
many interesting results have been found in mixed surfactants solutions, including the preparation of inorganic and organic nanoparticles and nanocomposites [16-18]. In our experiment, we found that by using the above systems, highly stable PPy dispersions were obtained and no sedimentation could be observed for at least 1 year. Therefore, we systemically studied the preparation for stable PPy dispersions by changing the conditions including the ratios of pyrrole monomer to the surfactant and PVP to SDS.

\section{Results and discussion}

\section{FT-IR spectra}

The FT-IR spectra of all the samples were collected through preparing films on silicon wafers. As can be seen from Fig. 1, the characteristic peaks of PPy are too weak to be easily found in Samples 1 to 5, which may have originated from two reasons: 1) the pyrrole was not well polymerized in the above five systems; 2) the films for measurement were not well formed due to the sedimentation of the dispersions (see Table 2). However, the dispersions without sedimentation show typical PPy peaks as follows: the peaks at 1540 and $1450 \mathrm{~cm}^{-1}$ are corresponding to symmetric and antisymmetric pyrrole ring-stretching modes; the strong peaks near 1194 and 930 $\mathrm{cm}^{-1}$ indicate the doping state of PPy and the peak at $1048 \mathrm{~cm}^{-1}$ presents the $\mathrm{C}-\mathrm{H}$ deformation vibration [15]. Besides, the peak located at $1650 \mathrm{~cm}^{-1}$ can be assigned to the stretching vibration of PVP molecule's $C=0$ group and two peaks at 2924 and $2856 \mathrm{~cm}^{-1}$ can be attributed to the stretching vibration mode of methylene (not shown here), indicating the surfactant molecules have been doped into the PPy structure.

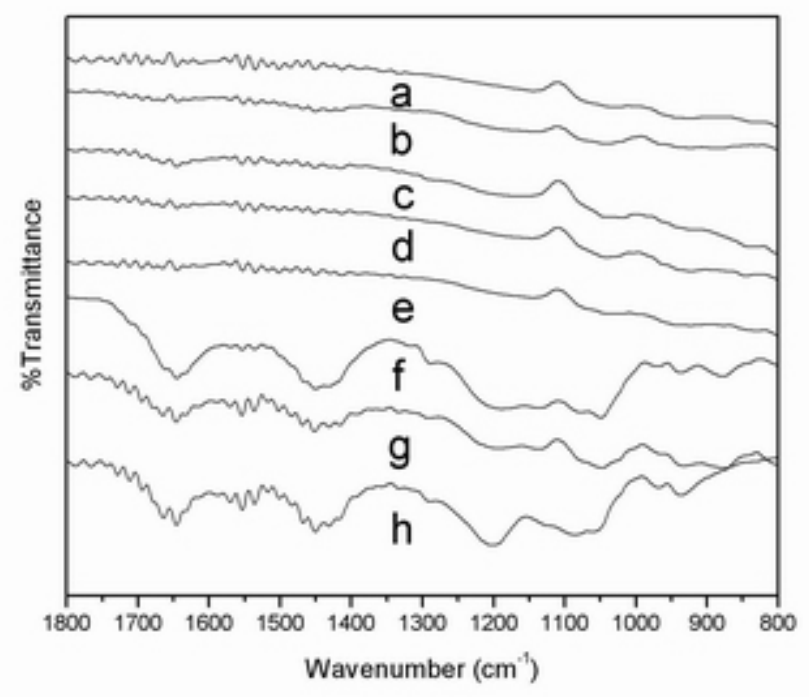

Fig. 1. FT-IR spectra of all the samples collected by forming films on silicon wafers (a to $\mathrm{h}$ refers Samples 1 to 8 in Table 2, respectively).

\section{Elemental composition}

Table 1 lists the elemental composition of PPy prepared in different conditions. The existence of $S$ indicates the anionic surfactant, SDS, is incorporated into the PPy structure in certain degree and the SDS may also exist in the samples with the 
adsorption interaction with PPy. For all the samples, the molar ratios of $\mathrm{N}$ to $\mathrm{S}$ are in the range from 2.26 (Sample 8) to 4.06 (Sample 3), indicating that for about three pyrrole rings one positive charge is created, which has to be balanced by the anion of oxidant molecule [19].

On the other hand, we can find the doping ratio of the PPy ranges from 0.49 (Sample 3 ) to 0.88 (Sample 8). When the molar ratios of pyrrole monomer to SDS is about or lower than 3, the sample shows relatively high doping level. This may have originated from the good doping effect of SDS when its content is relatively high, as discussed above.

Tab. 1. Elemental composition of different samples and their corresponding doping ratios and particle morphology.

\begin{tabular}{|c|c|c|c|c|c|c|c|}
\hline \multirow{2}{*}{ Sample $^{a}$} & \multirow{2}{*}{$N / S^{b}$} & \multicolumn{4}{|c|}{ Elemental analysis (wt.\%) } & \multirow{2}{*}{ Doping ratio $^{c}$} & \multirow{2}{*}{ Morphology } \\
\hline & & $\mathrm{C}$ & $\mathrm{H}$ & $\mathrm{N}$ & $\mathrm{S}$ & & \\
\hline 1 & 1 & 1 & 1 & 1 & 1 & I & Globular \\
\hline 2 & 2.88 & 66.22 & 6.11 & 9.67 & 8.56 & 0.77 & Globular \\
\hline 3 & 14.42 & 66.06 & 5.97 & 11.62 & 6.54 & 0.49 & Globular \\
\hline 4 & 5.77 & 65.16 & 5.93 & 11.63 & 7.59 & 0.57 & Globular \\
\hline 5 & 11.54 & 67.53 & 5.73 & 9.73 & 6.78 & 0.61 & Globular \\
\hline 6 & 2.88 & 65.92 & 5.84 & 9.71 & 8.38 & 0.75 & Irregular \\
\hline 7 & 2.88 & 65.86 & 6.06 & 9.82 & 8.49 & 0.76 & Irregular \\
\hline 8 & 1.44 & 66.51 & 5.72 & 8.92 & 9.01 & 0.88 & Irregular \\
\hline
\end{tabular}

\section{Morphology}

\section{Macroscopic observation}

Two typical samples were selected to show their morphology in this part, i.e., Samples 3 and 7 shown in Table 1.The film of PPy cast from Sample 3 gives apparent macroscopic phase separation, as can be seen from Fig. 2a. By contrast, the film cast from Sample 7 is homogeneous without macroscopic phase separation (Fig. 2b). It is well known that the surface smoothness of PPy films prepared from the aqueous solutions with addition of non-ionic surfactants is greatly improved in comparison with those prepared from the aqueous solutions in the absence of surfactants [20]. Meanwhile, the PPy films cannot be well formed only in the presence of anionic surfactants because of the strong ion strength existing in the PPy dispersion. Therefore, it is the common effects of PVP and SDS that make the film smooth and the dispersion stable. 


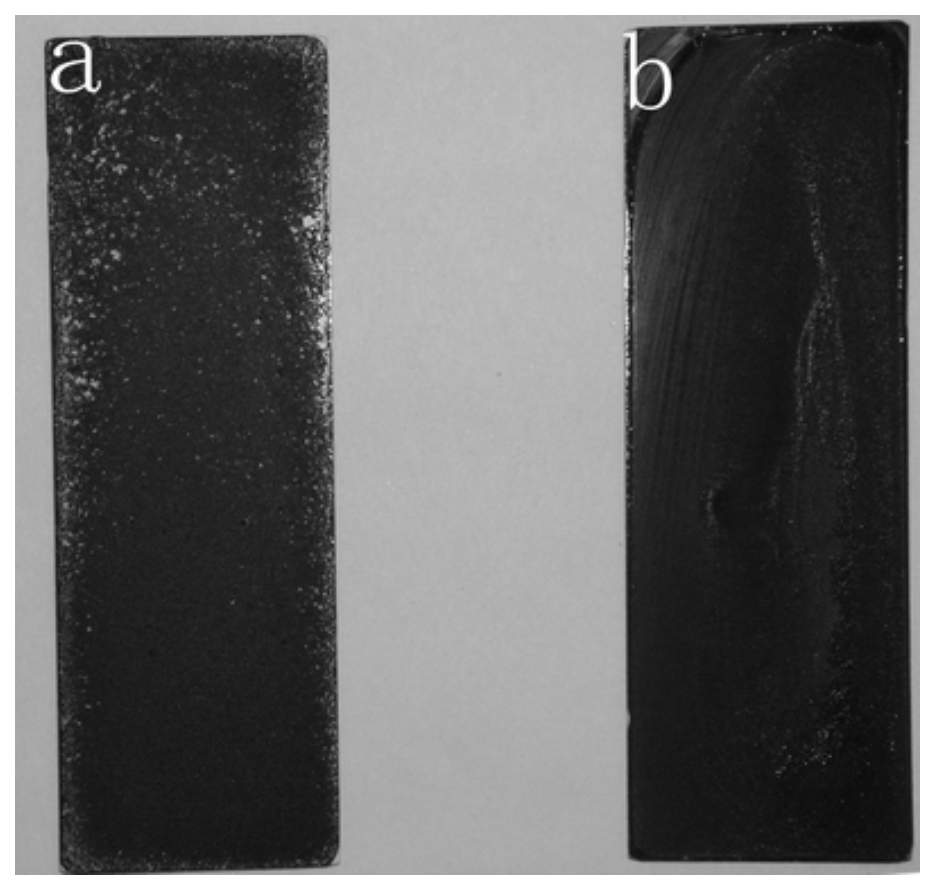

Fig. 2. Photos of the PPy films by dipping dispersions onto the glass substrates (a. Sample 3; b. Sample 7 listed in Table 2).

\section{Microscopic observation}

The SEM images of Sample 3 and 7 are presented in Fig. 3a and 3b, respectively. The globular particles with diameters averaged at about $200 \mathrm{~nm}$ can be seen from Fig. 3a, which is consistent with those obtained from the solution in the absence of surfactants. However, irregular morphology is found in Fig. 3b, showing Sample 7, the dispersion with high stability has different morphology with Sample 3 and other unstable dispersions. I. Díez et al. have reported the formation of PPy with unusual morphology in the presence of $\beta$-naphthalenesulfonic acid (NSA) and different fluorosurfactants [21]. They considered the resulting PPy was related to an in situ templating process, which involved the formation of the precipitate between the NSA, the fluorosurfactant and the monomer and the subsequent polymerization of the monomer on the surface of the complex. However, in our experiments, we did not observe the sedimentation before the oxidant was added into the solution. On the other hand, we find that the molar ratio of the monomer to the anionic surfactant greatly influences the morphology of the resulting PPy. When the molar ratios is equal to or lower than 2.88, the PPy shows irregular morphology. Otherwise, it is globular. As we have already discussed above, typically for three pyrrole rings one positive charge is created, which has to be balanced by the anion of the dopant [19]. Under that condition, the molar ratio of pyrrole to SDS should be equal to 3 . If the value is lower than 3 , free surfactant molecules exist in the solutions and they may have complex interaction between the PVP and the monomers, which perhaps leads to the formation of irregular morphological PPy. In our experiment, the SDS concentration is about or higher than $9 \mathrm{mM}$ except Sample 3, which is higher than the critical micelle concentration of SDS (about $8 \mathrm{mM}$ ). Therefore, the SDS can form micelles in the solutions and the interactions between the hydrophilic head grouping SDS and PVP chain may occur accompanying with adsorption between the three components including PPy, SDS and PVP. Meanwhile, if only SDS is used, no irregular morphological PPy can be observed even though the molar ratio of pyrrole 
to SDS is lower than 3, further indicating the common effects of SDS and PVP. It should be noted that from Fig. 3b, one can also find the existence of small particles of about $80 \mathrm{~nm}$ and we consider they are formed at the beginning of the polymerization and gradually assembled into the irregular morphology because of the interaction between the particles, SDS and PVP.
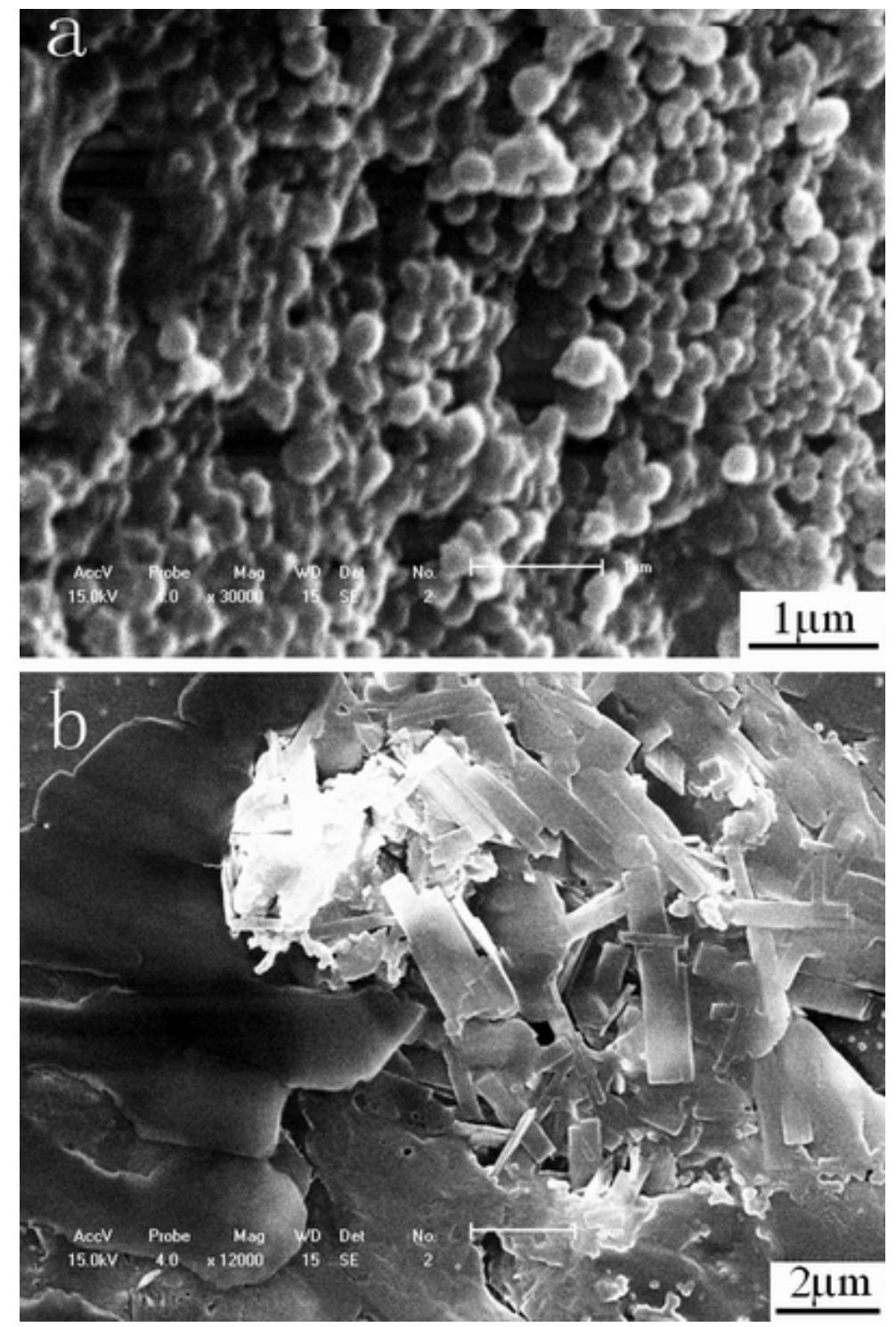

Fig. 3. SEM images of Sample 3 (a) and Sample 7 (b) listed in Table 2.

\section{Sedimentation}

As described above, when the molar ratio of the monomer to the anionic surfactant is about or lower than 3 , there are more free surfactant molecules that are not doped into the PPy structure in the system. Under this condition, except for the role to form irregular morphological product, these surfactants act as co-stabilizer in the dispersion owing to their interactions with the polymers in the presence of PVP. Therefore, as shown in Table 2, no sedimentation can be found at least after 1 year in Samples 6 to 8. However, the PPy dispersions prepared from sole SDS or PVP solutions or other systems with the molar ratio of pyrrole to SDS higher than 3 show sedimentation after a period of time. 


\section{Particle size and polydispersity index}

The particle sizes and the corresponding polydispersity indexes of the different samples are listed in Table 2. Because of their irregular morphology, no effective results could be obtained in Samples 6 to 8. One can find the PPy dispersion gives a particle size value of about $268 \mathrm{~nm}$ and the polydispersity index of 0.117 when it is prepared in sole PVP solution. However, the particle size of the PPy dispersion prepared in the sole SDS surfactant solution is about $70 \mathrm{~nm}$ and the polydispersity index is 0.400 . This may have originated from the difference between the anionic surfactant and PVP. When SDS is used in the preparation, the surfactant molecules form relatively stable micelles and provide proper places for the polymerization of pyrrole, which results in a small particle size. However, no stable micelles can be formed by PVP and no proper micro places can be constructed for the polymerization of pyrrole; therefore, the resulting PPy particle shows a larger size than that from the anionic surfactant. On the other hand, because the electrostatic interaction between the anionic surfactants and the polymers is stronger than that between PVP and the polymers, a larger polydispersity index is then obtained.

Tab. 2. Preparation conditions of PPy dispersions in the PVP/SDS mixed aqueous solution and their corresponding particle size $(d)$, polydispersity index and conductivity values $(\sigma)$.

\begin{tabular}{ccccccccc}
\hline Sample & $\begin{array}{c}\text { Pyrrole } \\
(\mathrm{M})\end{array}$ & $\begin{array}{c}\text { APS } \\
(\mathrm{M})\end{array}$ & $\begin{array}{c}\text { PVP } \\
(\mathrm{g})\end{array}$ & $\begin{array}{c}\text { SDS } \\
(\mathrm{g})\end{array}$ & $\begin{array}{c}\text { Sedi- } \\
\text { mentation }\end{array}$ & $\begin{array}{c}d \\
(\mathrm{~nm})\end{array}$ & $\begin{array}{c}\text { poly } \\
\text { index }\end{array}$ & $\begin{array}{c}\sigma \\
(\mathrm{mS} / \mathrm{cm})\end{array}$ \\
\hline 1 & 0.05 & 0.05 & 0.5 & $/$ & Yes & 268.3 & 0.117 & 14.72 \\
2 & 0.05 & 0.05 & $/$ & 0.5 & Yes & 70 & 0.400 & 14.93 \\
3 & 0.05 & 0.05 & 0.5 & 0.1 & Yes & 230.3 & 0.196 & 14.66 \\
4 & 0.05 & 0.05 & 0.5 & 0.25 & Yes & 210 & 0.225 & 14.38 \\
5 & 0.1 & 0.1 & 0.5 & 0.25 & Yes & 508 & 0.402 & 19.83 \\
6 & 0.025 & 0.025 & 0.5 & 0.25 & No & $/$ & $/$ & 9.31 \\
7 & 0.05 & 0.05 & 0.5 & 0.5 & No & $/$ & $/$ & 14.47 \\
8 & 0.05 & 0.05 & 0.5 & 1 & No & $/$ & $/$ & 14.88 \\
\hline
\end{tabular}

From Table 2, one can also get the following results: 1) When the content of the SDS in the mixed solutions is increased, the average diameters of the resulting PPy particles are decreased accordingly; 2) The particle sizes of the PPy in mixed solutions increase with the content of the pyrrole monomer. On one hand, SDS molecules can form relatively stable and small sized micelles compared with PVP molecules as discussed above, so the PPy shows smaller size when more SDS is added into the system; on the other hand, in the given polymerization places, when more pyrrole is added, larger size PPy particles are obtained.

\section{Conductivity}

The conductivity of the different samples is listed in Table 2. No apparent change can be observed except for Samples 5 and 6, which have different monomer 
concentration than others. Here, we consider that the conductivity of the dispersions is mainly influenced by two factors: 1) a higher concentration of pyrrole leads to a higher content of conducting PPy; 2) a higher ionic strength also results in a higher conductivity value.

\section{Conclusions}

Highly stable PPy dispersions were prepared using SDS and PVP as surfactant and polymeric stabilizer, respectively. The interaction between the two components and PPy, especially the free SDS molecules that are not doped into the PPy structure played an important role to form stable dispersions. The stable dispersions showed good film-forming characteristic and irregular morphology. By increasing the usage of SDS in the reaction system, the particle size of the resulting PPy was decreased accordingly because of the small polymerization places provided by the anionic surfactant. No sedimentation could be found in the stable dispersions at least after 1 year, showing the advantages of using PVP/SDS systems compared with using the single surfactant system.

\section{Experimental part}

\section{Materials}

Pyrrole was distilled under reduced pressure and other chemicals including APS, SDS, PVP (weight-average molecular weight $\approx 30,000$ ) were used as received without further purification. All regents were supplied by Sinopharm Chemical Reagent Co., LtdS, China.

\section{Preparation of PPy dispersion}

A typical experiment was done as follows: PVP and SDS were dissolved in deionized water with different mass ratios to form mixed solutions. Pyrrole was dissolved in the above solutions under stirring. APS in powder state was then directly added into the solutions, after which the solutions were stirred for another $1 \mathrm{~h}$. The so-obtained solutions were kept unstirred at room temperature and finally the color turned black, indicating PPy had been formed. Different preparation conditions are listed in Table 2 and the total volume of the solution was $100 \mathrm{~mL}$. No further treatments were carried out to remove the unreacted oxidant and monomer because we tried to optimize the preparation conditions and make easy and direct application of the dispersion.

\section{Characterization}

Fourier transformation infrared (FT-IR) spectrum of the sample was measured on an FTIR-8400s (Shimadzu) spectrometer in the transmission mode. A drop of PPy dispersion was dropped onto a piece of silicon wafer and a black film was then formed on the flake, which was used for the FTIR measurement of the PPy dispersion. Resolution of the measurements was equal to $4 \mathrm{~cm}^{-1}$ and silicon wafer was used as background.

The PPy composition was determined by elemental analysis using an elemental analyzer VarioEl.

10 drops of PPy dispersion was diluted by $5 \mathrm{~mL}$ of deionzed water. A drop of the above solution was dropped onto a piece of glass, dried in air and then a film was formed. The morphology of the film was observed using scanning electron 
microscopy (SEM, SSX-550, Shimadzu) with gold coating. The samples for the macro observation of the films were prepared without dilution of the dispersions.

A dynamic light scattering particle size distribution analyzer (ZETASIZER 3000HSA) was used for measurement of the PPy particle size distribution on diluting dispersion at $25^{\circ} \mathrm{C}$. For each value reported, at least three measurements were averaged.

Conductivity of dispersions was gathered with a standard conductometric cell composed of two blank platinum electrodes. DDS-11A meter operating at $1 \mathrm{kHz}$ frequency was used in the experimental set-up and the measurement was carried out at $25^{\circ} \mathrm{C}$. Due to the limitation of the instrument, all the dispersions were diluted to their $1 / 2$ concentration for measurement.

\section{Acknowledgements}

This work was supported by Science Foundation for Young Teachers of Northeast Normal University (NO 20060312).

\section{References}

[1] MacDiarmid A. G. Synth. Met. 1997, 84, 27.

[2] MacDiarmid A. G. Angew. Chem. Int. Ed. 2001, 40, 2581.

[3] Jang J.; Oh J. H. Langmuir 2004, 20, 8419.

[4] Zhang X.; Zhang J.; Song W.; Liu Z. J. Phys. Chem. B 2006, 110, 1158.

[5] Armes S. P.; Vincent B. J. Chem. Soc.; Chem. Commun. 1987, 288.

[6] Odegard R.; Skotheim T. A.; Lee H. S. J. Electrochem. Soc. 1991, 138, 2930.

[7] Qi Z.; Pickup P. G. Chem. Mater. 1997, 9, 2934.

[8] Digar M. L.; Bhattacharyya S. N.; Mandal B. M. Polymer 1994, 35, 377.

[9] Pich A.; Lu Y.; Adler H. J. P.; Schmidt T.; Arndt K. F. Polymer 2002, 43, 723.

[10] Mandal T. K.; Mandal B. M. Polymer 1995, 36, 1911.

[11] Saunders B. R.; Saunders J. M.; Mrkic J.; Dunlop E. H. Phys. Chem. Chem. Phys. 1999, 1, 1563.

[12] Flitton R.; Johal J.; Maeda S.; Armes S. P. J. Colloid. Interface. Sci. 1995, 173, 135.

[13] Lu Y.; Pich A.; Adler H. J. P. Macromol. Symp. 2004, 210, 411.

[14] DeArmitt C.; Armes S. P. Langmuir 1993, 9, 652.

[15] Xing S.; Zhao G. e-Polymers, 2007, no. 018.

[16] Zhao N.; Qi L. Adv. Mater. 2006, 18, 359.

[17] Xing S.; Zhao G. J. Appl. Polym. Sci. 2007, 104, 1987.

[18] Xing S.; Zhao G. Mater. Lett. 2007, 61, 2040.

[19] Xing S.; Zhao G. Polym. Bull. 2006, 57, 933.

[20] OmastováM.; TrchováM.; KovářováJ.; Stejskal J. Synth. Met. 2003, 138, 447.

[21] Díez I.; Tauer K.; Schulz B. Colloid. Polym. Sci. 2006, 284, 1431. 